\title{
Risk of aortic aneurysm and aortic dissection with the use of fluoroquinolones in Korea: a nested case-control study
}

\author{
Nayeong Son ${ }^{\dagger}$, Eunmi Choi ${ }^{\dagger}$, Soo Youn Chung, Soon Young Han and Bonggi Kim ${ }^{*}$
}

\begin{abstract}
Background: Recent studies have raised concern about the association of fluoroquinolones with an increased risk of aortic aneurysm and aortic dissection. We aimed to evaluate such risk in a Korean population.

Methods: We conducted a nested case-control study using data from the National Health Insurance Service collected from 2013 to 2017 in Korea. The study cohort included patients older than 40 years and excluded patients who had used fluoroquinolones or been diagnosed with aortic aneurysm, aortic dissection, or related diseases 1 year prior to the cohort entry date. We randomly matched four controls in the risk set with each case of aortic aneurysm and aortic dissection (same sex, age, and cohort entry date). We assessed the risk of aortic aneurysm and aortic dissection from fluoroquinolones and adjusted for potential confounders using a conditional logistic regression model.
\end{abstract}

Results: A total of 29,638 aortic aneurysm and aortic dissection patients were identified between 2014 and 2017. The use of fluoroquinolones within a year was associated with a 10\% increased risk of aortic aneurysm and aortic dissection (adjusted odds ratio: 1.10, 95\% Cl 1.07-1.14, $p<0.05$ ) compared with nonusers. The risk was higher in patients who had used fluoroquinolones within 60 days (adjusted odds ratio: 1.53, 95\% Cl 1.46-1.62, $p<0.05$ ). The risk of aortic aneurysm and aortic dissection positively correlated with the cumulative dose and duration of fluoroquinolone therapy $(p<0.001)$.

Conclusions: Our study provides real-world evidence of the risk of aortic aneurysm and aortic dissection from fluoroquinolones in Korea. Patients and medical professionals should be aware that fluoroquinolones can increase the risk of aortic aneurysm and aortic dissection, which may be acerbated by high dosage and duration of use.

Keywords: Fluoroquinolone, Aortic aneurysm, Aortic dissection, Drug safety, Pharmacovigilance, Adverse effect

*Correspondence: bgkim@drugsafe.or.kr

${ }^{+}$Nayeong Son and Eunmi Choi are co-first authors and contributed equally. All the authors take responsibility for all aspects of the reliability and freedom from bias of the data presented and their discussed interpretation

Korea Institute of Drug Safety and Risk Management, 6th FL, 30, Burim-ro

169beon-gil, Dongan-gu, Anyang-si, Gyeonggi-do, Republic of Korea

\section{Background}

Fluoroquinolones (FQs) are among the most widely used antibiotics in Korea, and their use has consistently increased to account for 9 to $11 \%$ of all antibiotic use [1]. Although FQs are powerful antibiotics with a wide antibacterial spectrum [2], they induce degradation of collagen and other structural components of the extracellular matrix by stimulating matrix metalloproteinases [3]. The possibility of excessive tissue breakdown by this mechanism has raised concern about the risk of adverse 
reactions, such as aortic aneurysm (AA) and aortic dissection $(\mathrm{AD})$.

In December 2018, the U.S. Food and Drug Administration warned that FQs can increase the occurrence of rare but serious ruptures or tears in the aorta. The warning included special caution for patients with a history of aneurysms, blockages, or hardening of the arteries, high blood pressure, or genetic conditions such as Marfan or Ehlers-Danlos syndrome and instructed patients to inform their health-care professional before starting a fluoroquinolone prescription [4]. Following that warning, the Ministry of Food and Drug Safety in Korea issued a safety letter warning about the potential association of fluoroquinolone use and the risk of AA/AD [5].

Many observational studies have suggested that fluoroquinolone use could be significantly associated with an increased risk of AA/AD [3, 6-9]. Recently, a systemic review and meta-analysis showed that fluoroquinolone use incurs a risk of developing three collagen-associated diseases, including AA/AD [10]. However, it has not yet been established whether fluoroquinolone use increases the risk of $\mathrm{AA} / \mathrm{AD}$ in the Korean population. This study aims to evaluate the association between fluoroquinolone use and the risk of AA/AD in the Korean population.

\section{Methods}

\section{Data source}

We conducted a nested case-control study using National Health Insurance Service (NHIS)-customized data (NHIS-2019-1-024). The NHIS database covers almost $98 \%$ of the total population in Korea. It contains patient demographic information such as sex, date of birth, date of death, and medical treatment records, including details of disease and prescriptions [11]. The authors declare no conflicts of interest with NHIS.

\section{Study population}

The study population comprised all patients aged 40 to 99 years 2014-2017 in the NHIS database. The date of 1 January 2014 was defined as the cohort entry date for patients aged 40 years or older in 2014. For patients aged less than 40 years in 2014, we established the cohort entry date as the first day of the year that the patient became 40 years old. We excluded 510,805 patients who:

- Had taken FQs more than once during the year prior to the cohort entry date

- Were diagnosed with AA/AD during the year prior to the cohort entry date

- Were diagnosed with underlying related diseases (atherosclerosis of the aorta, arteritis, aortitis, Lerche's syndrome, coarctation of the aorta, Marfan's syndrome, valve diseases, endocarditis, congenital malformations of valves, heart failure) (Additional file 1: Table S1) during the year prior to the cohort entry date.

\section{Case selection}

From the cohort, we identified 29,638 patients aged 40 years or older who had experienced AA/AD from 2014 to 2017 according to the definition of health outcomes of interest "Statistical analysis" section . Patients in the case group were observed from the cohort entry date to the index date, which was defined as the first date of diagnosis of AA/AD.

\section{Control selection}

After we stratified the case group based on age and sex, we created a risk set for each case using patients who were of the same sex and age as those with AA/AD and did not have a history of an AA/AD diagnosis. The size of the risk set was 20 times the sample size of each stratum. We randomly matched four controls in the risk set. Patients in the control group were observed from the cohort entry date to the index date of matched cases.

\section{Health outcomes of interest}

The outcome of the main analysis was defined as a diagnosis of AA/AD after entry to the cohort. Incident cases were defined as those who had received an ICD 10 code I71 (ICD 10 I71.0-I71.9) for all kinds of AA/AD. The outcome for the sensitivity analysis was redefined as a diagnosis of $\mathrm{AA} / \mathrm{AD}$ in addition to having received a laboratory test specific for AA/AD (abdominal/thoracic aortography, computed tomography $(\mathrm{CT})$, magnetic resonance imaging, ultrasonography, Doppler echocardiography, transesophageal/transthoracic echocardiography, abdominal vascular ultrasonography, or aorta Doppler ultrasonography) within 28 days prior to the diagnosis. The diagnosis and treatment of AA/AD were based on the ACCF/AHA/AATS/ACR/ASA/SCA/SCAI/SIR/STS/ SVM guideline in the general Korean hospitals [12]. The first date of diagnosis was defined as the index date for cases and matched controls.

\section{Exposure}

The exposure of interest was the use of a fluoroquinolone (balofloxacin, ciprofloxacin, enoxacin, gatifloxacin, gemifloxacin, levofloxacin, lomefloxacin, moxifloxacin, norfloxacin, ofloxacin, tosufloxacin, and zabofloxacin) in the year prior to the index date.

We categorized fluoroquinolone users as current, recent, or past users according to the time from the end of supply of the fluoroquinolone prescription to the index date. In this definition, 'termination of fluoroquinolone 
exposure' means the end of supply of the fluoroquinolone prescription. Current users were defined as patients who had terminated fluoroquinolone exposure within the 60 days prior to the index date. Recent users were defined as patients who had terminated fluoroquinolone exposure 61-120 days prior to the index date. Past users were defined as patients who had terminated fluoroquinolone exposure 121-365 days prior to the index date.

To investigate the effects of cumulative dose and duration of FQ exposure on the prevalence of $\mathrm{AA} / \mathrm{AD}$, we categorized fluoroquinolone users into three groups according to the quantiles of duration and into four groups according to the quantiles of cumulative dose. The duration was calculated as the sum of the total days of supply for each prescription in the year prior to the index date. The first and third quantiles of the cumulative days of supply were found to be 2 and 14 days, respectively. We represented the cumulative dose in the year prior to the index date in terms of the defined daily dose (DDD), as defined by the anatomical therapeutic chemical classification system. The first, second, and third quantiles of cumulative dose were found to be $4 \mathrm{DDD}$, 7.5 DDD, and 15 DDD, respectively.

The NHIS dataset included the Korean ingredient code of the drug, the date the prescription was written, the number of days of supply, and the quantity. We used these data to identify prescriptions for FQs and any concomitantly used drugs.

\section{Statistical analysis}

Pearson Chi-square tests and Fisher's exact test were used for the analysis of categorical variables. The odds ratios of the association between FQ use and AA/AD were calculated using multivariate conditional logistic regression analysis. We considered covariates known to be related to $\mathrm{AA} / \mathrm{AD}$ or fluoroquinolone use from previous studies and included them as confounders in the model [3, 6-9]. The covariates are listed in Table 1 . We also tested the tendency of AA/AD to occur with changes in timing, cumulative dose, and duration of FQ use using the Cochran-Armitage trend test. All data processing and statistical analyses were performed using SAS 9.4 and R 5.3.1 using two-sided tests, and a $p$ value of $<0.05$ was considered significant.

\section{Results}

\section{Demographic and clinical characteristics}

The final study population was composed of 148,190 patients, including 29,638 cases and 118,552 controls. Table 1 shows the baseline characteristics of the study population. This cohort comprised 92,645 male patients $(62.5 \%)$ and 55,545 female patients (37.5\%). More than half of the study population was $60-69$ years old (23.7\%) or 70-79 years old (32.0\%). Patients in the AA/AD case group had a higher prevalence of cerebrovascular disease and cardiovascular disorders such as arterial disease and ischemic heart disease. In the year prior to the index date. Patients in the AA/AD case group were more often users of angiotensin-converting enzyme inhibitors, antiarrhythmics, anticonvulsants, etc. from the cohort entry date to the index date and experienced more cardiac or aortic procedures and surgeries in the previous year.

\section{Association between AA/AD and FQ use}

During the observation period (1 year before the index date), 8562 cases (28.9\%) and 25,387 controls (21.4\%) received at least one prescription for FQs. Table 2 and Figure 1 show the results of the conditional logistic regression analysis. The adjusted odds ratio was 1.10 (95\% CI 1.07-1.14, $p<0.05$ ) during the 1-year observation period. However, the risk was substantially higher in current users (adjusted OR 1.53, 95\% CI 1.46-1.62, $p<0.05)$. FQ use did not have a significant association with AA/AD in recent users (adjusted OR 1.00, 95\% CI $0.93-1.07, p<0.05)$. The risk was even lower in past users (adjusted OR 0.92, 95\% CI 0.87-0.96, $p<0.05$ ).

The risk of AA/AD was studied according to the duration of exposure and the cumulative dose of FQs. In this study, 25\% of FQ users were exposed to FQs for 2 days or less. On the other hand, $25 \%$ of the FQ users were exposed to FQs for more than 14 days. Among them, $50 \%$ of the FQ users were exposed to FQs for between 3 days and 13 days. We used the same covariates as those adopted for the primary analysis. Patients who used FQs for less than three days had a lower risk of AA/AD than nonusers (adjusted OR 0.87, 95\% CI 0.82-0.92, $p<0.05$ ). However, the risk was significantly higher in patients who had used FQs for between three days and 13 days (adjusted OR 1.14, 95\% CI 1.09-1.19, $p<0.05$ ) and was highest in patients who used FQs for more than 14 days (adjusted OR 1.33, 95\% CI 1.26-1.40, $p<0.05$ ).

FQ users were categorized into four groups with regard to dose (low, mid-low, mid-high, or high) according to the quantiles of cumulative dose. Patients in the lowdose group had used FQs less than 4 DDDs during the observation period. Patients in the mid-low dose group and mid-high dose group had used 4 DDDs to 7.5 DDDs and 7.5 DDDs to 15 DDDs, respectively. Patients in the high-dose group had used more than 15 DDDs during the observation period. Compared with nonusers, the risk of AA/AD in the low-dose group ( $<4$ DDDs) was not significantly higher (adjusted OR $0.97,95 \%$ CI $0.89-1.04$, $p>0.05$ ). However, the risk was significantly higher in patients who had used FQs at more than 4 DDDs. Specifically, the adjusted odds ratio of AA/AD was 1.25 (95\% CI 1.16-1.34, $p<0.05)$ in the mid-low dose group, 1.29 
Table 1 Demographics and clinical characteristics of the study population

\begin{tabular}{|c|c|c|c|c|c|}
\hline & \multicolumn{2}{|c|}{ Case $(29,638)$} & \multicolumn{2}{|c|}{ Control $(118,552)$} & \multirow[t]{2}{*}{$p$ value* } \\
\hline & $\mathbf{N}$ & (\%) & $\mathbf{N}$ & (\%) & \\
\hline \multicolumn{6}{|l|}{ Sex } \\
\hline Male & 18,529 & 62.5 & 74,116 & 62.5 & 1 \\
\hline Female & 11,109 & 37.5 & 44,436 & 37.5 & \\
\hline \multicolumn{6}{|l|}{ Age (year) } \\
\hline $40-49$ & 2080 & 7 & 8320 & 7 & 1 \\
\hline $50-59$ & 4422 & 14.9 & 17,688 & 14.9 & \\
\hline $60-69$ & 7031 & 23.7 & 28,124 & 23.7 & \\
\hline 70-79 & 9479 & 32 & 37,916 & 32 & \\
\hline 80-89 & 5748 & 19.4 & 22,992 & 19.4 & \\
\hline $90+$ & 878 & 3 & 3512 & 3 & \\
\hline \multicolumn{6}{|l|}{ Underlying disease } \\
\hline Cerebrovascular disease & 3124 & 10.5 & 6418 & 5.4 & $<0.001$ \\
\hline Arterial disease & 8227 & 27.8 & 22,080 & 18.6 & $<0.001$ \\
\hline Ischemic heart disease & 7845 & 26.5 & 14,715 & 12.4 & $<0.001$ \\
\hline Cardiac valve disease & 884 & 3 & 444 & 0.4 & $<0.001$ \\
\hline Conduction disorder & 115 & 0.4 & 272 & 0.2 & $<0.001$ \\
\hline Heart failure or cardiomyopathy & 2723 & 9.2 & 4108 & 3.5 & $<0.001$ \\
\hline Chronic obstructive pulmonary disease & 10,626 & 35.9 & 31,726 & 26.8 & $<0.001$ \\
\hline Pneumonia & 3579 & 12.1 & 9047 & 7.6 & $<0.001$ \\
\hline Cancer & 3422 & 11.5 & 8462 & 7.1 & $<0.001$ \\
\hline Liver disease & 10,520 & 35.5 & 32,012 & 27 & $<0.001$ \\
\hline Renal disease & 1773 & 6 & 2902 & 2.4 & $<0.001$ \\
\hline Rheumatism & 2005 & 6.8 & 5720 & 4.8 & $<0.001$ \\
\hline Psychiatric disorder & 13,498 & 45.5 & 41,650 & 35.1 & $<0.001$ \\
\hline Diabetes & 9518 & 32.1 & 33,700 & 28.4 & $<0.001$ \\
\hline Hypertension & 19,716 & 66.5 & 60,520 & 51 & $<0.001$ \\
\hline Lipid disorder & 16,673 & 56.3 & 50,268 & 42.4 & $<0.001$ \\
\hline Trauma & 15,191 & 51.3 & 53,280 & 44.9 & $<0.001$ \\
\hline Obstructive sleep apnea & 73 & 0.2 & 190 & 0.2 & 0.002 \\
\hline Asthma & 6747 & 22.8 & 19,825 & 16.7 & $<0.001$ \\
\hline Obesity & 41 & 0.1 & 116 & 0.1 & 0.069 \\
\hline Seizure disorder & 1225 & 4.1 & 3015 & 2.5 & $<0.001$ \\
\hline Decubitus ulcer & 9 & 0 & 26 & 0 & 0.526 \\
\hline Infectious disease & 13,949 & 47.1 & 46,881 & 39.5 & $<0.001$ \\
\hline Hypothyrodism & 1729 & 5.8 & 5087 & 4.3 & $<0.001$ \\
\hline Inflammatory bowel disease & 3876 & 13.1 & 11,773 & 9.9 & $<0.001$ \\
\hline Urinary tract infection & 2287 & 7.7 & 5442 & 4.6 & $<0.001$ \\
\hline Ehlers-Danlos syndrome & 1 & 0 & 0 & 0 & 0.2 \\
\hline \multicolumn{6}{|l|}{ Charlson comorbidity Index } \\
\hline Mean(SD) & $2.67(2.41)$ & $1.86(2.07)$ & & & \\
\hline 0 & 5371 & 18.1 & 37,377 & 31.5 & $<0.001$ \\
\hline 1 & 5781 & 19.5 & 27,124 & 22.9 & \\
\hline 2 & 5425 & 18.3 & 19,785 & 16.7 & \\
\hline $3+$ & 13,061 & 44.1 & 34,266 & 28.9 & \\
\hline Myocardial infarction & 1377 & 4.6 & 1924 & 1.6 & $<0.001$ \\
\hline Congestive heart failure & 3731 & 12.6 & 6315 & 5.3 & $<0.001$ \\
\hline Peripheral vascular disease & 7376 & 24.9 & 20,782 & 17.5 & $<0.001$ \\
\hline Cerebrovascular disease & 6913 & 23.3 & 16,487 & 13.9 & $<0.001$ \\
\hline
\end{tabular}


Table 1 (continued)

\begin{tabular}{|c|c|c|c|c|c|}
\hline & Case $(2$ & & Control & 552) & $p$ value* \\
\hline & $\mathbf{N}$ & $(\%)$ & $\mathbf{N}$ & (\%) & \\
\hline Dementia & 3995 & 13.5 & 11,955 & 10.1 & $<0.001$ \\
\hline Chronic pulmonary diseases & 13,066 & 44.1 & 40,316 & 34 & $<0.001$ \\
\hline Connective tissue disease & 1827 & 6.2 & 5172 & 4.4 & $<0.001$ \\
\hline Peptic ulcer & 10,817 & 36.5 & 33,712 & 28.4 & $<0.001$ \\
\hline Mild liver diseases & 9697 & 32.7 & 29,509 & 24.9 & $<0.001$ \\
\hline Uncomplicated diabetes & 106 & 0.4 & 427 & 0.4 & 0.991 \\
\hline Diabetes complicated with retinopathy, neuropathy, renal disease & 2868 & 9.7 & 10,911 & 9.2 & 0.012 \\
\hline Hemiplegia & 936 & 3.2 & 2016 & 1.7 & $<0.001$ \\
\hline Moderate or severe renal diseases & 1773 & 6 & 2902 & 2.4 & $<0.001$ \\
\hline Nonmetastatic solid cancer, leukemia, lymphoma, multiple myeloma & 3288 & 11.1 & 8082 & 6.8 & $<0.001$ \\
\hline Moderate or severe liver diseases & 180 & 0.6 & 545 & 0.5 & 0.001 \\
\hline Metastatic solid cancer & 337 & 1.1 & 765 & 0.6 & $<0.001$ \\
\hline AIDS/HIV & 7 & 0 & 25 & 0 & 0.965 \\
\hline Medication use ${ }^{* *}$ & & & & & \\
\hline Angiotensin-converting enzyme inhibitors & 1119 & 3.8 & 2174 & 1.8 & $<0.001$ \\
\hline Antiarrhythmic & 13,410 & 45.2 & 34,164 & 28.8 & $<0.001$ \\
\hline Anticonvulsant & 3375 & 11.4 & 8889 & 7.5 & $<0.001$ \\
\hline Antidepressant & 5542 & 18.7 & 14,066 & 11.9 & $<0.001$ \\
\hline Immunodepressant & 8901 & 30 & 27,220 & 23 & $<0.001$ \\
\hline Anticoagulant & 20,898 & 70.5 & 68,727 & 58 & $<0.001$ \\
\hline$\beta$-blocker & 7664 & 25.9 & 15,373 & 13 & $<0.001$ \\
\hline Oral hypoglycemic agent & 3935 & 13.3 & 18,862 & 15.9 & $<0.001$ \\
\hline Benzodiazepine & 10,934 & 36.9 & 30,683 & 25.9 & $<0.001$ \\
\hline Calcium Channel Blockers & 13,194 & 44.5 & 38,216 & 32.2 & $<0.001$ \\
\hline corticosteroid & 16,632 & 56.1 & 56,174 & 47.4 & $<0.001$ \\
\hline Disease-modifying antirheumatic drugs & 618 & 2.1 & 1559 & 1.3 & $<0.001$ \\
\hline Insulin & 1136 & 3.8 & 2876 & 2.4 & $<0.001$ \\
\hline Loop diuretics & 3483 & 11.8 & 5350 & 4.5 & $<0.001$ \\
\hline Nonsteroidal anti-inflammatory drugs & 23,354 & 78.8 & 82,504 & 69.6 & $<0.001$ \\
\hline Antipsychotic & 7588 & 25.6 & 23,261 & 19.6 & $<0.001$ \\
\hline Peripheral vasodilators & 3425 & 11.6 & 4115 & 3.5 & $<0.001$ \\
\hline Lipid-lowering agent & 11,079 & 37.4 & 31,002 & 26.2 & $<0.001$ \\
\hline Parkinson medication & 1990 & 6.7 & 6220 & 5.2 & $<0.001$ \\
\hline Hydroxyzine & 2616 & 8.8 & 8049 & 6.8 & $<0.001$ \\
\hline Cardiac or aortic procedure/surgery & 287 & 1 & 251 & 0.2 & $<0.001$ \\
\hline
\end{tabular}

*The $p$ values are results from Chi-square or Fisher's exact tests

**Information on underlying disease were derived from data recorded prior to the index date and after cohort entry. Information on medication use were derived from data recorded in 1 year prior to the index date

Table 2 Results of conditional logistic regression analysis of the association between AA/AD and FQ use

\begin{tabular}{|c|c|c|c|c|c|c|c|c|}
\hline & \multicolumn{2}{|l|}{ Case } & \multicolumn{2}{|c|}{ Control } & \multicolumn{2}{|c|}{ Crude OR } & \multicolumn{2}{|c|}{ Adjusted OR* } \\
\hline & $\mathbf{N}$ & $\%$ & $\mathrm{~N}$ & $\%$ & OR & $95 \% \mathrm{Cl}$ & OR & $95 \% \mathrm{Cl}$ \\
\hline \multicolumn{9}{|c|}{ Main analysis } \\
\hline Nonusers & 21,076 & 71.1 & 93,165 & 78.6 & 1 & - & 1 & - \\
\hline Users & 8562 & 28.9 & 25,387 & 21.4 & $1.51^{* *}$ & $1.47-1.56$ & $1.10^{* *}$ & $1.07-1.14$ \\
\hline
\end{tabular}

*Adjusted for covariates presented in Table 1 (sex, age, underlying disease, Charlson comorbidity index, medication use, history of procedure/surgery) 


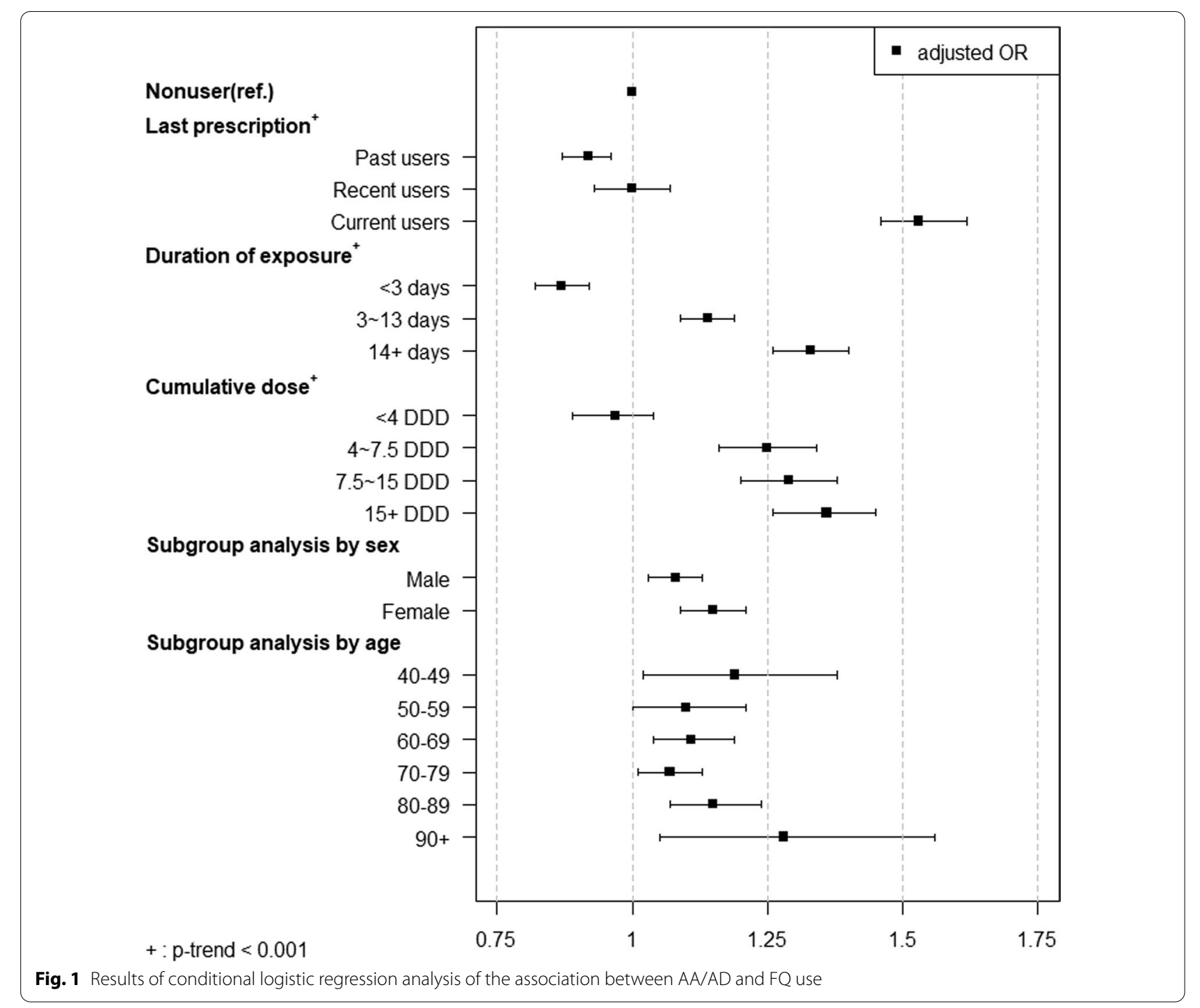

(95\% CI 1.20-1.38, $p<0.05$ ) in the mid-high dose group, and 1.36 (95\% CI 1.26-1.45) in the high dose group.

\section{Subgroup analysis and sensitivity analysis}

From the subgroup analysis by sex (Fig. 1), we found that the association between AA/AD and FQ use remained statistically significant in both the male and female subgroups. In particular, the risk was high in female patients (adjusted OR 1.15, 95\% CI 1.09-1.21, $p<0.05$ ) compared with female nonusers. When ages were grouped into 10-year bands, the association between AA/AD and FQ use remained statistically significant in every age group.

To verify the consistency of the results, we performed sensitivity analysis (Table 3 ) by changing the definition of AA/AD occurrence. The AA/AD occurrence in the primary analysis was identified using the ICD 10 code for AA/AD. For the sensitivity analysis, we changed the definition of an $\mathrm{AA} / \mathrm{AD}$ case to a diagnosis of $\mathrm{AA} / \mathrm{AD}$ in addition to having received a laboratory test specific for $\mathrm{AA} / \mathrm{AD}$ within the 28 days prior to the initial diagnosis of AA/AD. Among 29,648 AA/AD cases, 21,528 (72.6\%) received the laboratory test specific for $\mathrm{AA} /$ $\mathrm{AD}$ within the 28 days prior to the initial diagnosis of AA/AD. Among those 21,528 patients, 17,875 (83.0\%) were diagnosed with $\mathrm{AA} / \mathrm{AD}$ the day they took the tests. Abdominal/thoracic CT, aortography, and transthoracic echocardiography were found to have been the commonly performed procedures. The results remained consistent with the primary results under the new definition.

The risk of AA/AD by FQs was substantially higher in current users. The risk increased as the duration of exposure and cumulative dose increased. The association remained statistically significant in every subgroup 
Table 3 Results of sensitivity analysis of the association between AA/AD and FQ use

\begin{tabular}{|c|c|c|c|c|c|c|c|c|}
\hline & \multicolumn{2}{|l|}{ Case } & \multicolumn{2}{|c|}{ Controls } & \multicolumn{2}{|c|}{ Crude OR } & \multicolumn{2}{|c|}{ Adjusted OR* } \\
\hline & $\mathbf{N}$ & $\%$ & $\mathbf{N}$ & $\%$ & OR & $95 \% \mathrm{Cl}$ & OR & $95 \% \mathrm{Cl}$ \\
\hline \multicolumn{9}{|c|}{ Sensitivity analysis } \\
\hline Nonusers & 15,294 & 71.0 & 67,570 & 78.5 & 1 & - & 1 & - \\
\hline Users & 6234 & 29.0 & 18,542 & 21.5 & $1.51^{* *}$ & $1.46-1.56$ & $1.10^{* *}$ & $1.06-1.14$ \\
\hline
\end{tabular}

Cases that received laboratory tests specific for AA/AD within 28 days prior to the initial diagnosis of $A A / A D$ and their matched controls were included

*Adjusted for covariates presented in Table 1 (sex, age, underlying disease, Charlson comorbidity index, medication use, history of procedure/surgery)

${ }^{* *} p<0.05$

by sex and age. See Additional file 1: Table S2 for numeric results.

\section{Discussion}

In this study, FQ use showed a trend to be associated with an increased risk of AA/AD during the 1-year observation period, but the effect size was not remarkable. However, the risk of $\mathrm{AA} / \mathrm{AD}$ in current users of FQs was relatively considerable. This result is in line with preceding research in many ways. In an in vitro study that assessed the effect of FQs on MMP activities in human aortic smooth muscle cells, 48 hours of treatment with ciprofloxacin significantly increased total MMP activity. Observational studies using Taiwanese and Swedish databases also showed that the risk of AA/AD within 60 days after FQ use was significantly higher than that of nonusers $[3,7,8]$. In addition, a cohort study in Ontario, Canada and a signal analysis using U.S. FAERS data also indicated significant associations between FQ use and AA/AD [6, 9]. This trend is consistent with the results of a systematic literature review and meta-analysis conducted in 2019 [10]. In particular, Pasternak et al. [3] showed that the cumulative incidence of AA/AD increased significantly during the first 10 days after FQ use. Given these findings, further studies are needed to evaluate the risk in the early period of FQ use.

Studies that utilized the Taiwanese database $[7,8]$ reported that the risk of $\mathrm{AA} / \mathrm{AD}$ increased as the duration of drug use increased. In this study, the adjusted odds ratio of $\mathrm{AA} / \mathrm{AD}$ also increased as the cumulative duration of FQ use increased. In addition, while no prior study has determined the effect of the cumulative dose of $F Q s$ on the risk for $\mathrm{AA} / \mathrm{AD}$, this study showed that an increased cumulative dose of FQs could increase the risk for AA/AD. The dose-response relationship and duration-response relationship can be interpreted as considerable evidence of the causal relationship between FQ use and the occurrence of AA/
AD. Therefore, the patient's condition should be carefully monitored, keeping in mind that the risk of AA/ $\mathrm{AD}$ may increase as the cumulative dose or duration of FQ use increases.

Our study suggests some different results from the general understanding of $\mathrm{AA} / \mathrm{AD}$. In general, $\mathrm{AA} / \mathrm{AD}$ progresses slowly over several years, and men and old age are known as risk factors. However, we found that the risk of $\mathrm{AA} / \mathrm{AD}$ from FQ use was significant (1) in the early period of FQ use, (2) in female patients and male patients, and (3) in all age groups. In this research, the risk of $\mathrm{AA} / \mathrm{AD}$ was $8 \%$ higher in male FQ users and $15 \%$ higher in female FQ users than in nonusers of each sex. Although the risk difference between female patients and male patients was not statistically significant, it gives us a reasonable inference that female patients may have a higher risk of $\mathrm{FQ}$-induced $\mathrm{AA} / \mathrm{AD}$, contrary to general knowledge that the incidence of $\mathrm{AA} / \mathrm{AD}$ is higher in male patients. A previous study also showed that the risk was higher in female patients [7]. For age, the risk was significant in all age groups, but the differences between subgroups were not statistically significant. Given that the risk was higher in patients aged 70 or older in a previous study [7], we recommend that further research be undertaken to understand the risk factors for FQ-induced AA/ AD. The sensitivity analysis supported the robustness of the results, as they were very similar to the results before the definition of the study population was changed.

\section{Strengths and limitations}

As an indication of the strength of this research, it was conducted using the national health insurance claim data of all adults aged 40 or older in Korea during the five years from 2013 to 2017. The NHIS-customized data are well accumulated in the form of detailed medical activities and drugs, making it easy to generalize the research results, as nearly all domestic AA/AD patients were included in the study population. Additionally, we comprehensively considered various confounding factors, such as underlying diseases, medication use, and 
procedures and surgeries related to AA/AD. Moreover, we performed a sensitivity analysis by changing the definition of health outcomes of interest to minimize the effect of classification errors on the results. The preceding research results showed a $92 \%$ positive prediction for the identification of AA/AD cases when defining a group of cases considering both examination and diagnosis [7].

Our work clearly has some limitations. First, the results may have been affected by confounding indications. To reduce the bias from confounding indications, we excluded patients who had taken FQs during the year prior to the cohort entry date and included major indications of FQs as covariates in the adjusted model. However, the results may still have been affected by unmeasured underlying indications or the severity of the indication. The result must be carefully interpreted considering that patients who take FQs are possibly at higher risk of $\mathrm{AA} / \mathrm{AD}$ due to unmeasured underlying conditions, indications for the drug, and important risk factors such as smoking. We emphasize that this result should not be interpreted as explicit evidence for causal effects. It is clear that more studies would be necessary to determine whether there is a causal relationship.

Second, due to the nature of the claim data, it is difficult to pinpoint the exact timing of treatment and drug use, and it is not possible to analyze drug use, procedures, or surgeries that are not covered by NHIS. Third, socioeconomic and clinical confounding factors that could not be measured or predicted may have affected the results. For example, the difference in baseline characteristics of cases and controls can affect the results. To minimize the effect of known risk factors for AA/AD, we excluded patients with a history of AA/AD or related diseases during the year prior to the cohort entry date. However, some risk factors generally known to affect $\mathrm{AA} / \mathrm{AD}$, such as blood pressure, smoking status, and family history, were not considered in this study. In this sense, further studies are needed to evaluate the risk by patients' baseline health status and particular medical conditions, such as known risk factors for AA/AD.

Finally, the various clinical types and characteristics of $\mathrm{AA} / \mathrm{AD}$ were not analyzed because clinical information such as severity and detailed disease symptoms could not be fully determined by the diagnosis code alone. Thus, the results of this study are not appropriate for direct application to individuals, as patients may present with a variety of clinical characteristics. To overcome the potential bias introduced by confounding factors and the definition of exposure and health outcome of interest, we performed subgroup analysis, sensitivity analysis, and examined the dose-response relationship.

\section{Conclusion}

In this nested case-control study, we found that the use of FQs within a year was associated with a $10 \%$ increased risk of AA/AD in the Korean population. AA/ $\mathrm{AD}$ is a life-threatening disease accompanied by severe complications such as low blood pressure, shock, myocardial infarction, stroke, lower limb paralysis, and acute renal failure, which can lead to sudden death. In particular, early diagnosis and prompt treatment of abdominal AA/AD are critical, as $65 \%$ of patients die from cases of rupture [13]. Therefore, if patients feel symptoms such as chest pain in the early period of FQ use, even if the patient is not in the previously known risk group, medical professionals should suspect acute FQ-induced AA/AD, make a close diagnosis and consider changing or stopping the prescription. Moreover, if FQs are used in patients with already identified AA/ $\mathrm{AD}$, medical professionals should review the patient's history and carefully monitor them after drug administration, keeping in mind that FQs could increase the risk of $\mathrm{AA} / \mathrm{AD}$ and that the cumulative dose or duration of FQ use may affect the risk.

\section{Abbreviations}

AA: Aortic aneurysm; AD: Aortic dissection; DDD: Defined daily dose; FQs: Fluoroquinolones; NHIS: National Health Insurance Service.

\section{Supplementary Information}

The online version contains supplementary material available at https://doi. org/10.1186/s12872-022-02488-x.

Additional file 1: Table S1. ICD 10 code of AA/AD-related disease. Table S2. Results of conditional logistic regression analysis of the association between AA/AD and FQ use. Table S3. Frequency of underlying disease and mediation use in exposed and unexposed controls. Table S4. Association between AA/AD and FQ use in patients with cardiovascular diseases or indications of FQs.

\section{Acknowledgements}

We would like to thank the Benefits Strategy Department of the National Health Insurance Service for support.

\section{Authors' contributions}

NYS and EMC are co-first authors and contributed equally. NYS contributed to the conceptualization, methodology, software, statistical analysis, and writing of the original draft. EMC contributed to conceptualization, methodology, and writing - review \& editing. SYH and SYC contributed to supervision. BGK contributed to the supervision, project administration, writing, reviewing, and editing of the manuscript. All authors read and approved the final manuscript.

\section{Funding}

This research did not receive any specific grant from funding agencies in the public, commercial, or not-for-profit sectors.

\section{Availability of data and materials}

The data that support the findings of this study are available from the National Health Insurance Service in the Republic of Korea, but restrictions apply to the availability of these data, which were used under license for the current study and so are not publicly available. Data are, however, available from the authors 
upon reasonable request and with permission from the National Health Insurance Service.

\section{Declarations}

\section{Ethics approval and consent to participate}

Ethics approval for this study was obtained from the institutional review board of Korea Institute of Drug Safety and Risk Management, which waived informed consent (IRB approval number 2019-4). The study protocol conforms to the ethical guidelines of the 1975 Declaration of Helsinki.

\section{Consent for publication}

Not applicable.

\section{Competing interests}

The authors declare that they have no competing interests.

Received: 4 March 2021 Accepted: 31 January 2022

Published online: 13 February 2022

\section{References}

1. Kim Y, Park Y, Youk T, Lee S, Son Y. A study on the use of antibiotics in Korea and the resistance of major pathogens to antibiotics. NHIS IIsan hospital Report. 2016:20-001.

2. Redgrave LS, Sutton SB, Webber MA, Piddock LJ. Fluoroquinolone resistance: mechanisms, impact on bacteria, and role in evolutionary success. Trends Microbiol. 2014;22(8):438-45.

3. Pasternak B, Inghammar M, Svanström H. Fluoroquinolone use and risk of aortic aneurysm and dissection: nationwide cohort study. BMJ. 2018;360:k678.

4. Food and Drug Administration (FDA). Drug Safety Communication: FDA warns about increased risk of ruptures or tears in the aorta blood vessel with fluoroquinolone antibiotics in certain patients. 2018. https://www. fda.gov/Drugs/DrugSafety/ucm628753.htm. Accessed 14 Jan 2022.

5. Ministry of Food and Drug Safety (MFDS). A notification on the distribution of safety letters on fluoroquinolone antibiotics. https://www.mfds. go.kr/brd/m_545/view.do?seq=286\&srchFr=\&srchTo=\&srchWord=\% ED\%94\%8C\%EB\%A3\%A8\%EC\%98\%A4\%EB\%A1\%9C\%ED\%80\%B4\%EB\%

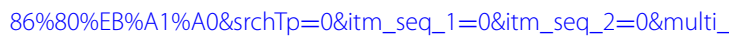

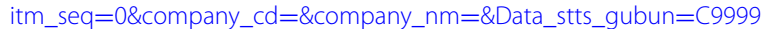
\&page=1. Accessed 14 Jan 2022.

6. Daneman N, Lu H, Redelmeier DA. Fluoroquinolones and collagen associated severe adverse events: a longitudinal cohort study. BMJ Open. 2015:5(11):e010077.

7. Lee C-C, Lee MG, Chen Y-S, Lee S-H, Chen Y-S, Chen S-C, et al. Risk of aortic dissection and aortic aneurysm in patients taking oral fluoroquinolone. JAMA Intern Med. 2015;175(11):1839-47.

8. Lee C-C, Lee MG, Hsieh R, Porta L, Lee W-C, Lee S-H, et al. Oral fluoroquinolone and the risk of aortic dissection. J Am Coll Cardiol. 2018;72(12):1369-78.

9. Meng L, Huang J, Jia Y, Huang H, Qiu F, Sun S. Assessing fluoroquinoloneassociated aortic aneurysm and dissection: data mining of the public version of the FDA adverse event reporting system. Int J Clin Pract. 2019;73(5):e13331.

10. Singh S, Nautiyal A. Aortic dissection and aortic aneurysms associated with fluoroquinolones: a systematic review and meta-analysis. Am J Med. 2017;130(12):1449-57.e9.

11. Lee J, Lee JS, Park S-H, Shin SA, Kim K. Cohort Profile: The National Health Insurance Service-National Sample Cohort (NHIS-NSC), South Korea. Int J Epidemiol. 2016;46(2):e15.

12. Foundation ACoC, Guidelines AHATFoP, Surgery AAfT, Radiology ACo, Association AS, Anesthesiologists SoC, et al. 2010 ACCF/AHA/AATS/ ACR/ASA/SCA/SCAI/SIR/STS/SVM guidelines for the diagnosis and management of patients with thoracic aortic disease. J Am Coll Cardiol. 2010;55(14):e27-129.

13. Sakalihasan $N$, Limet $R$, Defawe OD. Abdominal aortic aneurysm. Lancet. 2005;365(9470):1577-89.

\section{Publisher's Note}

Springer Nature remains neutral with regard to jurisdictional claims in published maps and institutional affiliations.
Ready to submit your research? Choose BMC and benefit from:

- fast, convenient online submission

- thorough peer review by experienced researchers in your field

- rapid publication on acceptance

- support for research data, including large and complex data types

- gold Open Access which fosters wider collaboration and increased citations

- maximum visibility for your research: over 100M website views per year

At BMC, research is always in progress.

Learn more biomedcentral.com/submissions 